Research Article

www.ijrap.net

\title{
EVALUATION OF EFFECTS OF YAVAKSHAR OINTMENT ON ABHYANTAR ARSHA (INTERNAL PILES)
}

Ukhalkar V. P.*

Associate Professor, Dept. of Shalya Tantra, Government Ayurved College, Nanded, Maharashtra, India

Received on: 23/05/13 Revised on: 27/06/13 Accepted on: 14/07/13

\author{
*Corresponding author \\ E-mail: ukhalkarvp@yahoo.com \\ DOI: $10.7897 / 2277-4343.04425$ \\ Published by Moksha Publishing House. Website www.mokshaph.com \\ All rights reserved.
}

\section{ABSTRACT}

A disease which anguishes patient's vital force or prana is called as Arsha according to Ayurveda. 'Arsha' is the gift of sedentary life style. One of its prime etiological factors is poor dietary habit which is unavoidable due to busy lifestyle. According to Sushruta the management of Arsha involves Aushadhi Chikitsa (conservative), Ksharkarma, Agnikarma and Shastrakarma (surgical). The 'Dhatuvaigunya' (Organic pathology local deformity) of anal structures requires local treatment. Ksharkarma is the treatment having more effect but needed intervention of another person for therapy. Concept of application of Kshar by patient himself was presumed and for this purpose the ointment of Kshar was used. The study was carried out in eight groups i.e. A, B, C, D, E, F, G, H and each group consists of about 25 to 30 patients. The patients of I II and III degree piles and P/R bleeding were included in this study. At the end of trial it was found that the application of Yava Kshar ointment of $10 \%$ having base Siktha Tailam was more effective than other ointments (i.e. Yava Kshar ointment 5\% base Siktha Tailam, Yava Kshar ointment 5\% base petroleum jelly, Yava Kshar ointment $10 \%$ base petroleum jelly, Faktu ointment, Trifgol powder, plane Siktha Tailam and plane Petroleum jelly) to reduced the P/R bleeding markedly in minimal period and to reduced the degree of haemorrhoids up to certain extent.

Keywords: Yava Kshar, Internal piles, Abhyantar Arsha, Kshar Karma, Yavakshar Ointment.

\section{INTRODUCTION}

Ayurved is a science of life and Shalya Tantra is its important branch which represents the surgical field. One of prime important disease from Ashtamahagada is 'Arsha' ${ }^{1}$. Ayurveda, the ancient science of medicine of India has detail information and description of Arsha. The word 'Arsha' is derived from the root 'Ru-gatau' after adding the suffix 'Asuna' which means 'to take life' ${ }^{2}$. It is an entity in which muscular projections (Mansakeel) troubles the patient like an enemy ${ }^{3}$. The common people call them piles. A pile (Pila- a ball) is derived from Latin. The aristocracy calls them haemorrhoids. The word haemorrhoid is derived from Greek. (Haema- blood, Rhoos- flowing) and the lay man call them mulvyadh / bawasir / komb etc. how does it matter as long as you can cure them ${ }^{4}$. Arsha have been known to mankind for a long time and are one of the commonest diseases to affect human beings. 'Arsha' is the gift of busy life style. One of its prime etiological factor is 'Mithyaahar- Vihar' ${ }^{5}$ and is unavoidable due to busy lifestyle. While working in Out Patient Department, it was observed that the percentage of patients having Abhyantar Gudarsha was increased. Hence, this problem was selected for scientific study. According to Ayurvedic and Modern text, so many mode of treatment are available for Abhyantar Gudarsha. The treatment can be classified in to surgical, para-surgical and medicinal management. But none of them is perfect due to their associated disadvantages. The management of Abhyantar Gudarsha is mentioned in Ayurvedic Samhita like Charak, Sushruta and Ashtang Sangraha etc. But Acharya Sushruta has described in detail all about Arsha in Sushruta Samhita. According to Acharya Sushruta the management of Abhyantar Gudarsha is of four types. Aushadhi Chikitsa (Conservative), Ksharkarma, Agnikarma, Shastrakarma (Surgical). The
'Dhatuvaigunya' (Organic pathology local deformity) of anal structures requires local treatment ${ }^{6}$. Ksharkarma is the treatment having more effect which was reported previously. Yava-Kshar was selected as a Kshar ingredient in previous study and it was done by using Yava-Kshar Pratisaran and this project was the extension of previous study ${ }^{7}$. But for Pratisaran of Kshar, intervention of doctor is necessary and compulsory. Hence it was decided to make the process convenient for patient to apply the Kshar at internal haemorrhoid and to avoid doctor's intervention for application of Kshar. Concept of application of Kshar by patient was presumed and for this purpose the Ointment of Kshar was preferred. Here the study was carried out, to prove the effect of application of Yava Kshar ointment at internal haemorrhoid (Abhyantar Gudarsha). The Yava Kshar ointment was prepared with two different concentration i.e. $5 \%$ and $10 \%$ and also two different base were selected one was natural and other was synthetic i.e. Siktha Tailam and Petroleum Jelly respectively. During pilot study 5\% and $10 \%$ composition was used to verify results and it was continued for final study. Due to minimum concentration of Yava Kshar the unwanted corrosion to normal mucosa was avoided.

\section{Aim}

To provide an effective local treatment, as local application in the form of Malhar (ointment) having the direct local effect in the management of Arsha (Internal Piles).

\section{Objectives}

- To modify the ancient mode of treatment in the scientific era.

- Evaluation of effects Yava-Kshar ointment application on Internal piles (Abhyantar Gudarsha) 


\section{MATERIALS AND METHODS Preparation of Yava Kshar}

Yava (Hordeum vulgare Linn.) sown in land and Yava was cultivated. It was grown fully approximately in 3 months. Then the soil was irrigated up to 8 days. After that the plants were pulled out along with roots. Plants were dried completely in sunlight in few days. The ash was obtained by burning the all dried Yava plants. That ash was dissolved in 6 times of water and maintained for one day. Next day that water was filtered by 12 folded fine cloth. The filtered water had appearance and smell like a cows urine. The filtered water was evaporated completely by heating it. Lastly the whitish Yava Kshar was obtained at bottom of container ${ }^{8}$.

\section{Preparation of Ointment}

Preparation of Yava Kshar ointment was carried out at Government Ayurved Pharmacy, Nanded, Maharashtra, India. By using base Petroleum jelly and Siktha Tailam9 (Bees wax + Sesame Oil) different compositions were prepared by mixture and grinder machine. Compositions were as follow

1. Petroleum Jelly + Yava Kshar 5\% w/w

2. Petroleum jelly + Yava Kshar $10 \%$ w/w

3. Siktha Tailam + Yava Kshar 5\% w/w

4. Siktha Tailam + Yava Kshar $10 \% \mathrm{w} / \mathrm{w}$

Above said compositions were filled in different tubes using manual filling machine. Each tube was filled by 30 $\mathrm{g}$ of ointment separately.

\section{Physicochemical Analysis}

Analysis of Yava Kshar was done in Food and Drug Administration (FDA) approved laboratory yields good results. (Table 1 and 2)

\section{Clinical Study}

Before the initiation of the study, the study protocol and related documents were reviewed and approved by Institutional Ethics Committee at Government Ayurved College, Nanded, Maharashtra, India.

Institutional Ethical Clearance (GACN/IEC/25-35/09 Dated-15/06/2009)

Patients (age group, 21 to 70years) attending the Outpatient Clinic at Government Ayurved Hospital, Nanded, Maharashtra, India and meeting all the inclusion criteria were recruited in the trial. A written consent was obtained by counseling the patients of piles for participation in the study.

\section{Selection Criteria for Patients}

- Patients of piles of both sexes were included in this study.

- The study groups of age group between 21 years to 70 years age.

- The patients of I, II and III degree piles included in this study.

- All cases were fresh and had not received any local treatment.

Rejection Criteria for Patients

- The patients of piles having previous history of haemorrhoidectomy were rejected.

- Patients of piles which were diabetic, malignant. AIDS, Koch's with fistula or having portal obstruction was rejected.

- Patients having $\mathrm{Hb}$ below $10 \mathrm{~g} \%$ were rejected.

- Pregnant woman was not included in this study.

- Patients suffering from chronic toxicity were excluded from this study.

The study was carried out in eight groups i.e. A, B, C, D, E, F, G, H and each group consists of about 25 to 30 patients. The process of Yava Kshar Pratisaran was modified according to nature of study. Patient was advised Mrudu-Virechan in the form of bowel regulator (Trifgol powder) and Seitz bath regularly. The patient was advised to apply Yava Kshar ointment with the help of applicator provided with ointment tube twice daily.

\section{A Group}

Treated with local Yava Kshar 5\% ointment (base petroleum jelly) with dietetic regime and bowel regulator

\section{B Group}

Treated with local Yava Kshar 5\% ointment (base Siktha Tailam) with dietetic regime and bowel regulator

\section{Group}

Treated with local Yava-Kshar 10\% ointment (base petroleum jelly) with dietetic regime and bowel regulator

\section{D group}

Treated with local Yava Kshar 10\% ointment (base Siktha Tailam) with dietetic regime and bowel regulator

\section{E group}

Treated with popular allopathic brand name 'Faktu ointment' (Contents policruselin 5\%+Cinchocaine 1\%) Manufactured by and GLAT company with dietetic regime and bowel regulator. This group taken as a comparative.

\section{F Group}

Received only bowel regulator adopting standard dietetic regime

\section{G Group}

Treated with local petroleum jelly application with dietetic regime and bowel regulator

\section{H group}

Treated with local Siktha Tailam application with dietetic regime and bowel regulator

\section{Criteria for Assessment}

\section{Degree of Hemorrhoid}

I - Hemorrhoids projecting slightly in lumen of anal canal, when veins are congested at defecation.

II - Hemorrhoids prolapsed out of the anus on straining, but return spontaneously to the anal canal when motion has been passed and the defection has ceased.

III - Haemorrhoids prolapse but don't reduce spontaneously and remain prolapsed afterwards and have 
to be replaced digitally.

IV - Completely irreducible haemorrhoids usually are long standing and acquire a component of skin.

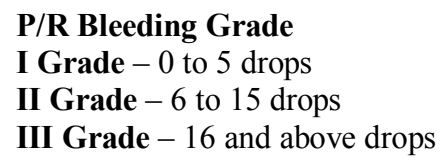

A special clinical record form (C.R.F.) was prepared to record the findings. Every patient was observed at regular follow up on $0^{\text {th }}, 3^{\text {rd }}, 10^{\text {th }}, 17^{\text {th }}$ and $24^{\text {th }}$ day. For visual recording the regular photographs of selected patients from each group were taken for observing the local changes at internal haemorrhoid. Routine investigations of all patients and some specific investigations were carried out as and when required.

\section{Dietetic Regime was used}

- Diet rich in fibers was advised.

- Avoid Non Vegetarian Diet.

- Avoiding Spicy Diet.

\section{Statistical Analysis}

The confidence limit was fixed at $95 \%$ and the level of significance was at $5 \%$. Paired and unpaired ' $t$ ' test was applied for objective parameter and chi square test was applied for subjective parameter. Analysis of $\mathrm{P} / \mathrm{R}$ bleeding in each group was done by applying ' $\mathrm{t}$ ' test to difference of $\mathrm{P} / \mathrm{R}$ bleeding between each follow up and then before and after treatment.

\section{Observations}

In this study 219 volunteers were selected randomly and these volunteers were grouped under various groups mentioned above i.e. minimum 26 patients per group. The important demographic findings of these groups are compiled in Table 3 to 9 .

\section{Statistical Analysis and Interpretation}

The confidence limit was fixed at $95 \%$ and the level of significance was at $5 \%$. Paired and unpaired ' $t$ ' test was applied for objective parameter and chi square test was applied for subjective parameter and ANOVA test was applied for comparison of effect of all ointments. Analysis of $\mathrm{P} / \mathrm{R}$ bleeding in each group was done by applying ' $t$ ' test to difference of $P / R$ bleeding between each follow up and then before and after treatment. Maximum number of patients was from age group of 25 to 40years. Maximum numbers of patients were consuming mix and spicy diet. The distribution of patient in all groups according to above said effective factors was equal. The observed difference was statistically insignificant. Hence all groups were from the same population and comparable at baseline. In all groups incidence of $3^{\text {rd }}$ grade $P / R$ bleeding was maximum and incidence of $1^{\text {st }}$ grade $\mathrm{P} / \mathrm{R}$ bleeding was minimum. This may be due to negligence of patients in less severity. It was observed that in all groups incidence of $2^{\text {nd }}$ degree hemorrhoids were maximum due to inclusive criteria of groups. It was least in $1^{\text {st }}$ degree hemorrhoids may be due to negligence of patients. (Table 10)
From Table 10 it can be concluded that the distribution of patient in all groups according to above said effective factors was equal. The observed difference was the statistically insignificant. Hence all groups were from the same population and comparable at baseline.

Group Yava Kshar ointment 5\% base Petroleum Jelly Above observation and significance test shown that difference of $\mathrm{P} / \mathrm{R}$ bleeding before and after treatment was highly significant. Hence it can be said that change occurred may be due to treatment. (Table 11)

\section{Group Yava Kshar ointment 5\% base Siktha Tailam}

Above observation and significance test shown that difference of $\mathrm{P} / \mathrm{R}$ bleeding before and after treatment was highly significant. Hence it can be said that change occurred may be due to treatment. (Table 12)

\section{Group Yava Kshar ointment $\mathbf{1 0 \%}$ base Petroleum Jelly}

Above observation and significance test shown that difference of $\mathrm{P} / \mathrm{R}$ bleeding before and after treatment was highly significant. Hence it can be said that change occurred may be due to treatment. (Table 13)

Group Yava Kshar ointment $10 \%$ base Siktha Tailam Above observation and significance test shown that difference of $\mathrm{P} / \mathrm{R}$ bleeding before and after treatment was highly significant. Hence it can be said that change occurred may be due to treatment. (Table 14)

\section{Faktu Ointment}

Above observation and significance test shown that difference of $\mathrm{P} / \mathrm{R}$ bleeding before and after treatment was highly significant. Hence it can be said that change occurred may be due to treatment. (Table 15)

\section{Trifgol (Table 16)}

\section{Siktha Tailam (Table 17)}

\section{Petroleum Jelly (Table 18)}

From Table no. 16 to 18 it can be concluded that there was no difference occurred in these groups. Treatments were mostly ineffective.

From the Table 19 ( $\mathrm{a}$ and $\mathrm{b}$ ) it can be concluded that $\mathrm{F}$ value for degree of freedom 2,81 is 3.15 and 4.98 for 0.05 and 0.001 level of significance. As $F(2,81)$ calculated is more than table value of $\mathrm{F}(2,81)$ there is significant difference in the three treatments i.e. Yavakshar ointment 5\% (base Siktha Tailam), Yava Kshar ointment 10\% (base petroleum jelly), Yava Kshar ointment $10 \%$ (base Siktha Tailam).

From the Table 20 ( $\mathrm{a}$ and $\mathrm{b}$ ) in can be concluded that $\mathrm{F}$ value for degree of freedom 5, 158 is 2.21 and 3.02 for 0.05 and 0.001 level of significance. As F $(5,158)$ calculated is less than table value of $F(5,158)$ there is insignificant difference in the six treatments.

Table 21 shows that the result of treatment of group D was best i.e. maximum numbers of patients were cured and all other relieved. 
Ukhalkar V. P/Int. J. Res. Ayurveda Pharm. 4(4), Jul-Aug 2013

Table 1: Results of Physicochemical analysis of Yava Kshar

\begin{tabular}{|c|c|}
\hline Test & Result \\
\hline Moisture & $38.42 \%$ \\
\hline Total Ash & $24.30 \%$ \\
\hline Acid insoluble in Dil. HCl & $3.7 \%$ \\
\hline Specific Gravity & 1.2156 \\
\hline Potassium & $198 \mathrm{mg} / 100 \mathrm{gm}$ \\
\hline Sodium & $23 \mathrm{mg} / 100 \mathrm{gm}$ \\
\hline Fluoride & $15 \mathrm{mg} / 100 \mathrm{gm}$ \\
\hline pH Value & 10.60 \\
\hline
\end{tabular}

Table 2: Results of Physicochemical analysis of Yava Kshar ointment prepared by various concentrations

\begin{tabular}{|c|c|c|c|c|}
\hline Name of Ointment & Pile Sikt 5\% & Pile Gel 5\% & Pile Sikt 10\% & Pile Gel 10\% \\
\hline Moisture & $0.14 \%$ & $0.13 \%$ & $0.11 \%$ & $0.12 \%$ \\
\hline Total Ash & $1.20 \%$ & $1.19 \%$ & $2.42 \%$ & $2.27 \%$ \\
\hline Acid insoluble in Dil. HCl & $0.17 \%$ & $0.14 \%$ & $0.38 \%$ & $0.25 \%$ \\
\hline Specific Gravity & 0.9379 & 0.9312 & 0.9352 & 0.956 \\
\hline Potassium & $10 \mathrm{mg} / 100 \mathrm{gm}$ & $9 \mathrm{mg} / 100 \mathrm{gm}$ & $21 \mathrm{mg} / 100 \mathrm{gm}$ & $19 \mathrm{mg} / 100 \mathrm{gm}$ \\
\hline Sodium & $2 \mathrm{mg} / 100 \mathrm{gm}$ & $1 \mathrm{mg} / 100 \mathrm{gm}$ & $3 \mathrm{mg} / 100 \mathrm{gm}$ & $2 \mathrm{mg} / 100 \mathrm{gm}$ \\
\hline Fluoride & $1 \mathrm{mg} / 100 \mathrm{gm}$ & $1 \mathrm{mg} / 100 \mathrm{gm}$ & $2 \mathrm{mg} / 100 \mathrm{gm}$ & $2 \mathrm{mg} / 100 \mathrm{gm}$ \\
\hline Iodine Value & 6.96 & 7.1 & 6.56 & 6.32 \\
\hline Melting point & $34^{0} \mathrm{C}$ & $33.4^{0} \mathrm{C}$ & $34.6{ }^{0} \mathrm{C}$ & $35^{0} \mathrm{C}$ \\
\hline Saponification value & 255.46 & 258.8 & 261.35 & 259.67 \\
\hline Acid Value & 2.62 & 2.52 & 2.35 & 2.41 \\
\hline Unsaponificable matter & $0.59 \%$ & $0.62 \%$ & $0.68 \%$ & $0.65 \%$ \\
\hline
\end{tabular}

Table 3: Sex wise incidences of patients in all groups

\begin{tabular}{|c|c|c|c|}
\hline Groups & Male & Female & Total \\
\hline A & 23 & 3 & 26 \\
\hline B & 25 & 3 & 28 \\
\hline C & 24 & 3 & 27 \\
\hline D & 25 & 4 & 29 \\
\hline E & 21 & 5 & 26 \\
\hline F & 25 & 3 & 28 \\
\hline G & 24 & 4 & 28 \\
\hline H & 21 & 6 & 27 \\
\hline Total & 188 & 31 & 219 \\
\hline \multicolumn{2}{|r|}{} & $\mathrm{X}^{2}=0.89424$ & $\mathrm{P}=\mathrm{P}>0.05$ \\
\hline
\end{tabular}

Table 4: Age wise incidences of patients in all groups

\begin{tabular}{|c|c|c|c|c|}
\hline Groups & $\mathbf{2 5 - 4 0}$ & $\mathbf{4 0 - 5 5}$ & $\mathbf{5 5 - 7 0}$ & Total \\
\hline A & 13 & 9 & 4 & 26 \\
\hline B & 9 & 11 & 8 & 28 \\
\hline C & 14 & 8 & 5 & 27 \\
\hline D & 19 & 9 & 1 & 29 \\
\hline E & 9 & 10 & 7 & 26 \\
\hline F & 11 & 13 & 4 & 28 \\
\hline G & 12 & 13 & 3 & 28 \\
\hline H & 9 & 14 & 4 & 27 \\
\hline Total & 96 & 87 & 36 & 219 \\
\hline \multicolumn{6}{|l}{$*$ Age in years } & $\mathrm{X}^{2}=0.25732$ & $\mathrm{P}=\mathrm{P}>0.05$ \\
\hline
\end{tabular}

Table 5: Diet wise incidences of patients in all groups

\begin{tabular}{|c|c|c|c|c|c|c|c|c|c|c|c|}
\hline \multirow[t]{2}{*}{ Diet } & \multicolumn{8}{|c|}{ Groups } & \multirow[t]{2}{*}{ Total } & \multirow[t]{2}{*}{$\mathrm{X}^{2}$ value } & \multirow{2}{*}{$\begin{array}{c}\mathbf{p} \\
\text { value }\end{array}$} \\
\hline & $\mathrm{A}$ & $\mathrm{B}$ & $\mathrm{C}$ & $\mathrm{D}$ & $\mathrm{E}$ & $\mathrm{F}$ & $\mathrm{G}$ & $\mathrm{H}$ & & & \\
\hline Mix & 25 & 27 & 26 & 27 & 26 & 28 & 27 & 27 & 213 & 0.71847 & $\mathrm{p}>0.05$ \\
\hline Veg & 1 & 1 & 1 & 2 & 0 & 0 & 1 & 0 & 06 & & \\
\hline Spicy & 26 & 28 & 27 & 27 & 26 & 27 & 27 & 26 & 214 & 0.54715 & $\mathrm{p}>0.05$ \\
\hline Non spicy & 0 & 0 & 0 & 0 & 2 & 1 & 1 & 1 & 05 & & \\
\hline
\end{tabular}

Table 6: Degree of $P / R$ bleeding wise incidences of patients in all groups

\begin{tabular}{|c|c|c|c|c|}
\hline Groups & $\mathbf{3}^{\text {rd }}$ & $\mathbf{2}^{\text {nd }}$ & $\mathbf{1}^{\text {st }}$ & Total \\
\hline A & 17 & 9 & 0 & 26 \\
\hline B & 21 & 7 & 0 & 28 \\
\hline C & 22 & 5 & 0 & 27 \\
\hline D & 23 & 5 & 1 & 29 \\
\hline E & 19 & 7 & 0 & 26 \\
\hline F & 21 & 7 & 0 & 28 \\
\hline G & 21 & 7 & 0 & 28 \\
\hline H & 20 & 7 & 0 & 27 \\
\hline Total & 164 & 54 & 1 & 219 \\
\hline \multicolumn{5}{|l}{} \\
\hline
\end{tabular}

Table 7: Degree of hemorrhoid wise incidence of patient in all groups

\begin{tabular}{|c|c|c|c|c|}
\hline Groups & $\mathbf{3}^{\text {rd }}$ & $\mathbf{2}^{\text {nd }}$ & $\mathbf{1}^{\text {st }}$ & Total \\
\hline A & 3 & 23 & 0 & 26 \\
\hline B & 1 & 27 & 0 & 28 \\
\hline C & 2 & 25 & 0 & 27 \\
\hline D & 4 & 25 & 0 & 29 \\
\hline E & 1 & 25 & 0 & 26 \\
\hline F & 0 & 28 & 0 & 28 \\
\hline G & 2 & 26 & 0 & 28 \\
\hline H & 4 & 22 & 1 & 27 \\
\hline Total & 17 & 201 & 1 & 219 \\
\hline \multicolumn{6}{|r}{} & $\mathrm{X}^{2}=0.39132$ & $\mathrm{P}=\mathrm{P}>0.05$ \\
\hline
\end{tabular}


Ukhalkar V. P/Int. J. Res. Ayurveda Pharm. 4(4), Jul-Aug 2013

Table 8: showing the mean of PR bleeding on each follow up in different Groups

\begin{tabular}{|c|c|c|c|c|c|c|c|c|c|c|}
\hline \multirow{2}{*}{ Group } & \multicolumn{2}{|c|}{$\mathbf{0}^{\text {th }}$ day } & \multicolumn{2}{c|}{$\mathbf{3}^{\text {rd }}$ day } & \multicolumn{2}{c|}{$\mathbf{1 0}^{\text {th }}$ day } & \multicolumn{2}{c|}{$\mathbf{1 7}^{\text {th }}$ day } & \multicolumn{2}{c|}{$\mathbf{2 4}^{\text {th }}$ day } \\
\cline { 2 - 12 } & Mean & S.D. & Mean & S.D. & Mean & S.D. & Mean & S.D. & Mean & S.D. \\
\hline A & 2.654 & 0.485 & 2.077 & 0.628 & 0.808 & 0.801 & 0.038 & 0.196 & 0 & 0 \\
\hline B & 2.75 & 0.441 & 0.607 & 0.786 & 0.179 & 0.39 & 0.036 & 0.189 & 0 & 0 \\
\hline C & 2.759 & 0.511 & 0.31 & 0.66 & 0.069 & 0.371 & 0.034 & 0.186 & 0 & 0 \\
\hline D & 2.815 & 0.396 & 0.704 & 0.775 & 0.296 & 0.542 & 0.037 & 0.192 & 0 & 0 \\
\hline E & 2.731 & 0.452 & 2.615 & 0.496 & 1.346 & 0.629 & 0.654 & 0.689 & 0.385 & 0.496 \\
\hline F & 2.75 & 0.441 & 2.643 & 0.488 & 2.5 & 0.509 & 2.321 & 0.612 & 2.036 & 0.744 \\
\hline G & 2.741 & 0.447 & 2.63 & 0.565 & 2.704 & 0.542 & 2.667 & 0.555 & 2.63 & 0.565 \\
\hline H & 2.75 & 0.441 & 2.679 & 0.476 & 2.786 & 0.418 & 2.75 & 0.441 & 2.714 & 0.46 \\
\hline
\end{tabular}

Table 9: Showing the mean degree of haemorrhoid on each follow up in different groups

\begin{tabular}{|c|c|c|c|c|c|c|c|c|c|c|}
\hline \multirow{2}{*}{ Group } & \multicolumn{2}{|c|}{$\mathbf{0}^{\text {th }}$ day } & \multicolumn{2}{|c|}{$\mathbf{3}^{\text {rd }}$ day } & \multicolumn{2}{|c|}{$\mathbf{1 0}^{\text {th }}$ day } & \multicolumn{2}{|c|}{$\mathbf{1 7}^{\text {th }}$ day } & \multicolumn{2}{|c|}{$\mathbf{4}^{\text {th }}$ day } \\
\cline { 2 - 12 } & Mean & S.D. & Mean & S.D. & Mean & S.D. & Mean & S.D. & Mean & S.D. \\
\hline A & 2.115 & 0.326 & 2.115 & 0.326 & 2.115 & 0.326 & 2.115 & 0.326 & 2.115 & 0.326 \\
\hline B & 2.036 & 0.189 & 2.036 & 0.189 & 2.036 & 0.189 & 2.036 & 0.189 & 1.857 & 0.448 \\
\hline C & 2.074 & 0.267 & 2.074 & 0.267 & 2.074 & 0.267 & 2.074 & 0.267 & 1.815 & 0.396 \\
\hline D & 2.138 & 0.351 & 2.138 & 0.351 & 2.138 & 0.351 & 2.138 & 0.351 & 1.69 & 0.604 \\
\hline E & 2.038 & 0.196 & 2.038 & 0.196 & 2.038 & 0.196 & 2.038 & 0.196 & 2.038 & 0.196 \\
\hline F & 2.00 & 00 & 2.00 & 00 & 2.00 & 00 & 2.00 & 00 & 2.00 & 00 \\
\hline G & 2.111 & 0.424 & 2.111 & 0.424 & 2.111 & 0.424 & 2.111 & 0.424 & 2.111 & 0.424 \\
\hline H & 2.071 & 0.262 & 2.071 & 0.262 & 2.071 & 0.262 & 2.071 & 0.262 & 2.071 & 0.262 \\
\hline
\end{tabular}

Table 10: Analysis of Incidence

\begin{tabular}{|c|c|c|}
\hline Attribute & $\mathbf{X}^{\mathbf{2}}$ & $\mathbf{p}$ \\
\hline Sex & 0.89424 & $\mathrm{P}>0.05$ \\
\hline Age & 0.25732 & $\mathrm{P}>0.05$ \\
\hline Diet (mix/ veg.) & 0.71847 & $\mathrm{P}>0.05$ \\
\hline (Spicy / No spicy) & 0.54751 & $\mathrm{P}>0.05$ \\
\hline Degree of P/R bleeding & 0.81024 & $\mathrm{P}>0.05$ \\
\hline Degree of piles & 0.39132 & $\mathrm{P}>0.05$ \\
\hline
\end{tabular}

Table 11: Showing the significance of difference in PR Bleeding on each follow up and after complete treatment

\begin{tabular}{|c|c|c|c|c|c|}
\hline & $0^{\text {th }}$ day & $3^{\text {rd }}$ day & $10^{\text {th }}$ day & $17^{\text {th }}$ day & BT-AT \\
\hline Mean of difference & 0.577 & 1.269 & 0.769 & 0.038 & 2.077 \\
\hline S.D. & 0.578 & 0.724 & 0.765 & 0.196 & 0.628 \\
\hline S.E. & 0.113355 & 0.141988 & 0.150029 & 0.038439 & 0.123161 \\
\hline T & 5.09 & 8.937 & 5.126 & 0.989 & 16.864 \\
\hline P & $\mathrm{P}<0.05$ & $\mathrm{P}<0.05$ & $\mathrm{P}<0.05$ & $\mathrm{P}<0.05$ & $\mathrm{P}<0.05$ \\
\hline & $\mathrm{P}<0.001$ & $\mathrm{P}<0.001$ & $\mathrm{P}<0.001$ & $\mathrm{P}<0.001$ & $\mathrm{P}<0.001$ \\
\hline
\end{tabular}

Table 12: Showing the significance of difference in PR Bleeding on each follow up and after complete treatment

\begin{tabular}{|c|c|c|c|c|c|}
\hline & $\mathbf{0}^{\text {th }}-\mathbf{3}^{\text {rd }}$ & $\mathbf{3}^{\text {rd }}-\mathbf{1 0}^{\text {th }}$ & $\mathbf{1 0}^{\text {th }}-\mathbf{1 7}^{\text {th }}$ & $\mathbf{1 7}^{\text {th }}-\mathbf{2 4}^{\text {th }}$ & $\mathbf{B T}-\mathbf{A T}$ \\
\hline Mean of difference & 2.143 & 0.429 & 0.095 & 0.048 & 2.714 \\
\hline S.D. & 0.891 & 0.507 & 0.301 & 0.218 & 0.463 \\
\hline S.E. & 0.168 & 0.096 & 0.057 & 0.041 & 0.087 \\
\hline $\mathbf{t}$ & 12.756 & 4.469 & 1.667 & 1.171 & 31.195 \\
\hline $\mathbf{p}$ & $\mathrm{P}<0.05$ & $\mathrm{P}<0.05$ & $\mathrm{P}>0.05$ & $\mathrm{P}>0.05$ & $\mathrm{P}<0.05$ \\
\hline & $\mathrm{P}<0.001$ & $\mathrm{P}<0.001$ & $\mathrm{P}<0.001$ & $\mathrm{P}<0.001$ & $\mathrm{P}<0.001$ \\
\hline
\end{tabular}

Table 13: Showing the significance of difference in PR Bleeding on each follow up and after complete treatment

\begin{tabular}{|c|c|c|c|c|c|}
\hline & $\mathbf{0}^{\text {th }}-\mathbf{3}^{\text {rd }}$ & $\mathbf{3}^{\text {rd }}-\mathbf{1 0}^{\text {th }}$ & $\mathbf{1 0}^{\text {th }}-\mathbf{1 7}^{\text {th }}$ & $\mathbf{1 7}^{\text {th }}-\mathbf{2 4}^{\text {th }}$ & $\mathbf{B T}-\mathbf{A T}$ \\
\hline Mean of difference & 2.111 & 0.407 & 0.259 & 0.037 & 2.815 \\
\hline S.D. & 0.847 & 0.501 & 0.447 & 0.192 & 0.396 \\
\hline S.E. & 0.163005 & 0.096417 & 0.086025 & 0.03695 & 0.07621 \\
\hline $\mathbf{t}$ & 12.951 & 4.221 & 3.011 & 1.001 & 36.937 \\
\hline $\mathbf{p}$ & $\mathrm{P}<0.05$ & $\mathrm{P}<0.05$ & $\mathrm{P}<0.05$ & $\mathrm{P}>0.05$ & $\mathrm{P}<0.05$ \\
& $\mathrm{P}<0.001$ & $\mathrm{P}<0.001$ & $\mathrm{P}>0.001$ & $\mathrm{P}>0.001$ & $\mathrm{P}<0.001$ \\
\hline & $\mathrm{P}<0.001$ & $\mathrm{P}<0.001$ & $\mathrm{P}<0.001$ & $\mathrm{P}<0.001$ & $\mathrm{P}<0.001$ \\
\hline
\end{tabular}


Ukhalkar V. P/Int. J. Res. Ayurveda Pharm. 4(4), Jul-Aug 2013

Table 14: Showing the significance of difference in $P / R$ bleeding on each follow up and after complete treatment

\begin{tabular}{|c|c|c|c|c|c|}
\hline & $\mathbf{0}^{\text {th }}-\mathbf{3}^{\text {rd }}$ & $\mathbf{3}^{\text {rd }}-\mathbf{1 0}^{\text {th }}$ & $\mathbf{1 0}^{\text {th }}-\mathbf{1 7}^{\text {th }}$ & $\mathbf{1 7}^{\text {th }}-\mathbf{2 4}^{\text {th }}$ & $\mathbf{B T}^{-\mathbf{A T}}$ \\
\hline Mean of difference & 2.448 & 0.241 & 0.034 & 0.034 & 2.759 \\
\hline S.D. & 0.87 & 0.435 & 0.186 & 0.186 & 0.511 \\
\hline S.E. & 0.162 & 0.081 & 0.035 & 0.035 & 0.095 \\
\hline $\mathbf{t}$ & 15.111 & 2.975 & 0.971 & 0.971 & 29.042 \\
\hline $\mathbf{p}$ & $\mathrm{P}<0.05$ & $\mathrm{P}<0.05$ & $\mathrm{P}>0.05$ & $\mathrm{P}>0.05$ & $\mathrm{P}<0.05$ \\
\hline \multicolumn{2}{|r|}{} & $\mathrm{P}<0.001$ & $\mathrm{P}<0.001$ & $\mathrm{P}<0.001$ & $\mathrm{P}<0.001$ \\
\hline
\end{tabular}

Table 15: Showing the significance of difference in PR Bleeding on each follow up and after complete treatment

\begin{tabular}{|c|c|c|c|c|c|}
\hline & $\mathbf{0}^{\text {th }}-\mathbf{3}^{\text {rd }}$ & $\mathbf{3}^{\text {rd }}-\mathbf{1 0}^{\text {th }}$ & $\mathbf{1 0}^{\text {th }}-\mathbf{1 7}^{\text {th }}$ & $\mathbf{1 7}^{\text {th }}-\mathbf{2 4}^{\text {th }}$ & BT $-\mathbf{A T}$ \\
\hline Mean of difference & 0.115 & 1.269 & 0.692 & 0.269 & 2.231 \\
\hline S.D. & 0.326 & 0.667 & 0.549 & 0.452 & 0.587 \\
\hline S.E. & 0.063934 & 0.130809 & 0.107668 & 0.088644 & 0.11512 \\
\hline $\mathbf{t}$ & 1.799 & 9.701 & 6.427 & 3.035 & 19.38 \\
\hline $\mathbf{p}$ & $\mathrm{P}>0.05$ & $\mathrm{P}<0.05$ & $\mathrm{P}<0.05$ & $\mathrm{P}<0.05$ & $\mathrm{P}<0.05$ \\
\hline & $\mathrm{P}>0.001$ & $\mathrm{P}<0.001$ & $\mathrm{P}<0.001$ & $\mathrm{P}>0.001$ & $\mathrm{P}<0.001$ \\
\hline
\end{tabular}

Table 16: Showing the significance of difference in PR Bleeding on each follow up and after complete treatment

\begin{tabular}{|c|c|c|c|c|c|}
\hline & $\mathbf{0}^{\text {th }}-\mathbf{3}^{\text {rd }}$ & $\mathbf{3}^{\text {rd }}-\mathbf{1 0}^{\text {th }}$ & $\mathbf{1 0}^{\text {th }}-\mathbf{1 7}^{\text {th }}$ & $\mathbf{1 7}^{\text {th }}-\mathbf{2 4}^{\text {th }}$ & BT $-\mathbf{A T}$ \\
\hline Mean of difference & 0.107 & 0.143 & 0.179 & 0.286 & 0.607 \\
\hline S.D. & 0.315 & 0.356 & 0.67 & 0.763 & 0.786 \\
\hline S.E. & 0.059529 & 0.067278 & 0.126618 & 0.144193 & 0.14854 \\
\hline $\mathbf{t}$ & 1.797 & 2.126 & 1.414 & 1.983 & 4.086 \\
\hline $\mathbf{p}$ & $\mathrm{P}>0.05$ & $\mathrm{P}<0.05$ & $\mathrm{P}>0.05$ & $\mathrm{P}>0.05$ & $\mathrm{P}<0.05$ \\
\hline & $\mathrm{P}>0.001$ & $\mathrm{P}>0.001$ & $\mathrm{P}>0.001$ & $\mathrm{P}>0.001$ & $\mathrm{P}<0.001$ \\
\hline
\end{tabular}

Table 17: Showing the significance of difference in $P / R$ bleeding on each follow up and after complete treatment

\begin{tabular}{|c|c|c|c|c|c|}
\hline & $\mathbf{0}^{\text {th }}-\mathbf{3}^{\text {rd }}$ & $\mathbf{3}^{\text {rd }}-\mathbf{1 0}^{\text {th }}$ & $\mathbf{1 0}^{\text {th }}-\mathbf{1 7}^{\text {th }}$ & $\mathbf{1 7}^{\text {th }}-\mathbf{2 4}^{\text {th }}$ & BT $-\mathbf{A T}$ \\
\hline Mean of difference & 0.111 & -0.074 & 0.037 & 0.037 & 0 \\
\hline S.D. & 0.32 & 0.267 & 0.192 & 0.192 & 0.392 \\
\hline S.E. & 0.061584 & 0.051384 & 0.03695 & 0.03695 & 0.07544 \\
\hline $\mathbf{t}$ & 1.802 & -1.44 & 1.001 & 1.001 & 0 \\
\hline $\mathbf{p}$ & $\mathrm{P}>0.05$ & $\mathrm{P}>0.05$ & $\mathrm{P}>0.05$ & $\mathrm{P}>0.05$ & $\mathrm{P}>0.05$ \\
\hline \multicolumn{2}{|r|}{} & $\mathrm{P}>0.001$ & $\mathrm{P}>0.001$ & $\mathrm{P}>0.001$ & $\mathrm{P}>0.001$ \\
\hline
\end{tabular}

Table 18: Showing the significance of difference in PR Bleeding on each follow up and after complete treatment

\begin{tabular}{|c|c|c|c|c|c|}
\hline & $\mathbf{0}^{\text {th }}-\mathbf{3}^{\text {rd }}$ & $\mathbf{3}^{\text {rd }}-\mathbf{1 0}^{\text {th }}$ & $\mathbf{1 0}^{\text {th }}-\mathbf{1 7}^{\text {th }}$ & $\mathbf{1 7}^{\text {th }}-\mathbf{2 4}^{\text {th }}$ & $\mathbf{B T}-\mathbf{A T}$ \\
\hline Mean of difference & 0.071 & -0.107 & 0.036 & 0.036 & -0.036 \\
\hline S.D. & 0.262 & 0.416 & 0.429 & 0.744 & 0.637 \\
\hline S.E. & 0.049513 & 0.078617 & 0.081073 & 0.140603 & 0.120382 \\
\hline $\mathbf{t}$ & 1.434 & -1.361 & 0.444 & 0.256 & -0.299 \\
\hline $\mathbf{p}$ & $\mathrm{P}>0.05$ & $\mathrm{P}>0.05$ & $\mathrm{P}>0.05$ & $\mathrm{P}>0.05$ & $\mathrm{P}>0.05$ \\
\hline & $\mathrm{P}>0.001$ & $\mathrm{P}>0.001$ & $\mathrm{P}>0.001$ & $\mathrm{P}>0.001$ & $\mathrm{P}>0.001$ \\
\hline
\end{tabular}

Table 19 (a): Differences in Degree of Hemorrhoid due to Treatment

\begin{tabular}{|c|c|c|c|c|c|c|c|c|}
\hline & $\begin{array}{c}\text { Petro } \\
\mathbf{5 \%}\end{array}$ & $\begin{array}{c}\text { Siktha } \\
\mathbf{5 \%}\end{array}$ & $\begin{array}{c}\text { Siktha } \\
\mathbf{1 0} \%\end{array}$ & $\begin{array}{c}\text { Petro } \\
\mathbf{1 0} \%\end{array}$ & Faktu & Trifgol & PetroGel & $\begin{array}{c}\text { Siktha } \\
\text { Tailam }\end{array}$ \\
\hline Mean & 0 & 0.179 & 0.448 & 0.259 & 0 & 0 & 0 & 0 \\
\hline SD & 0 & 0.39 & 0.506 & 0.447 & 0 & 0 & 0 & 0 \\
\hline SE & 0 & 0.074 & 0.094 & 0.086 & 0 & 0 & 0 & 0 \\
\hline $\mathrm{t}$ & 0 & 2.419 & 4.766 & 3.012 & 0 & 0 & 0 & 0 \\
\hline $\mathrm{P}$ & $\mathrm{P}>0.05$ & $\mathrm{P}<0.05$ & $\mathrm{P}<0.05$ & $\mathrm{P}<0.05$ & $\mathrm{P}>0.05$ & $\mathrm{P}>0.05$ & $\mathrm{P}>0.05$ & $\mathrm{P}>0.05$ \\
\hline \multicolumn{7}{|c|}{ Grand Mean $=0.298$ Degree of freedom (Total) $=83$} \\
\hline
\end{tabular}

Table 19(b): Difference in Degree of Hemorrhoid due to Treatment

\begin{tabular}{|c|c|c|c|c|}
\hline $\begin{array}{c}\text { Source of } \\
\text { Variation }\end{array}$ & $\begin{array}{c}\text { Degrees of } \\
\text { freedom }\end{array}$ & $\begin{array}{c}\text { Sum of } \\
\text { Squares }\end{array}$ & $\begin{array}{c}\text { Mean sum } \\
\text { of square }\end{array}$ & F \\
\hline $\mathrm{T} / \mathrm{T}$ & 2 & 8.535 & 4.268 & 38.8 \\
\hline Error & 81 & 8.935 & 0.11 & \\
\hline Total & 83 & 17.47 & & \\
\hline
\end{tabular}


Table 20 (a): Difference in $P / R$ Bleeding due to Treatment

\begin{tabular}{|c|c|c|c|c|c|c|c|c|}
\hline & $\begin{array}{c}\text { Petro } \\
\mathbf{5 \%} \\
\end{array}$ & $\begin{array}{c}\text { Siktha } \\
\mathbf{5 \%} \\
\end{array}$ & $\begin{array}{c}\text { Siktha } \\
10 \% \\
\end{array}$ & $\begin{array}{c}\text { Petro } \\
10 \%\end{array}$ & Faktu & Trifgol & Petro Gel & $\begin{array}{c}\text { Siktha } \\
\text { Tailam } \\
\end{array}$ \\
\hline Mean & 2.077 & 2.714 & 2.759 & 2.815 & 2.231 & 0.607 & -0.036 & \\
\hline SD & 0.628 & 0.463 & 0.511 & 0.396 & 0.587 & 0.786 & 0.637 & 0.392 \\
\hline SE & 0.123 & 0.087 & 0.095 & 0.0762 & 0.1151 & 0.1485 & 0.1203 & 0.0754 \\
\hline$t$ & 16.864 & 31.195 & 29.042 & 36.937 & 19.38 & 4.086 & -0.299 & 0 \\
\hline \multirow[t]{2}{*}{$\mathbf{P}$} & $\mathrm{P}<0.05$ & $\mathrm{P}<0.05$ & $\mathrm{P}<0.05$ & $\mathrm{P}<0.05$ & $\mathrm{P}<0.05$ & $\mathrm{P}<0.05$ & $\mathrm{P}>0.05$ & $\mathrm{P}>0.05$ \\
\hline & $\mathrm{P}<0.001$ & $\mathrm{P}<0.001$ & $\mathrm{P}<0.001$ & $\mathrm{P}<0.001$ & $\mathrm{P}<0.001$ & $\mathrm{P}<0.001$ & $\mathrm{P}>0.001$ & $\mathrm{P}>0.001$ \\
\hline
\end{tabular}

Table 20 (b): Difference in P/R Bleeding due to Treatment

\begin{tabular}{|c|c|c|c|c|}
\hline $\begin{array}{c}\text { Source of } \\
\text { Variation }\end{array}$ & $\begin{array}{c}\text { Degrees of } \\
\text { freedom }\end{array}$ & $\begin{array}{c}\text { Sum of } \\
\text { Squares }\end{array}$ & $\begin{array}{c}\text { Mean sum } \\
\text { of square }\end{array}$ & F \\
\hline $\mathrm{T} / \mathrm{T}$ & 5 & 898.225 & 179.645 & -37.7326 \\
\hline Error & 158 & -752.176 & -4.761 & - \\
\hline Total & 163 & 146.049 & - & - \\
\hline
\end{tabular}

Table 21: Results of treatment of patients in all groups

\begin{tabular}{|c|c|c|c|c|}
\hline Groups & Cured & Relived & Not cured & Total \\
\hline A & 0 & 26 & 0 & 26 \\
\hline B & 5 & 23 & 0 & 28 \\
\hline C & 7 & 20 & 0 & 27 \\
\hline D & 14 & 15 & 0 & 29 \\
\hline E & 0 & 17 & 9 & 26 \\
\hline F & 0 & 0 & 28 & 28 \\
\hline G & 0 & 0 & 28 & 28 \\
\hline H & 0 & 0 & 27 & 27 \\
\hline Total & 26 & 101 & 92 & 219 \\
\hline \multicolumn{4}{r}{$\mathrm{X}^{2}=236.1}$, & $\mathrm{P}<0.05$ \\
\end{tabular}

\section{DISSCUSION}

Haemorrhoid (pile) is the most frequently observed anal pathology. This disease has been recognized and treated since antiquity. Much more modern information is available on the pathogenesis of haemorrhoid. The basic pathology appears to be centered on the absence of valves in the haemorrhoidal vessels followed by lack of supportive structures around the vein and precipitating or provoking factors like increased intra abdominal and intrarectal pressure, improper dietary habitat with addictions. Normal anatomical structure involved in haemorrhoids present in every one after birth, which are thought to play some part in anal continence. Internal haemorrhoids are considered as disease after the symptoms like bleeding, protrusion, inflammation and thrombosis.

The treatment must be aimed at

- Symptomatic relief and

- The correction of anatomical deformity.

Above mentioned treatment are achieved by means of conservation or surgical methods. Now a day various non surgical methods are an alternative to surgical ones. They aim at tissue fixation with or without tissue destruction like sclerotherapy, photocoagulation/IRC (Infra Red Coagulation) and Barron's Band Ligation. Increasing number of therapies themselves proves that there is no universally acceptable technique in the management of haemorrhoids. Since, the haemorrhoid problem is not a threat to life excepting few conditions; the least invasive treatment is most desirable. Ksharkarma is the treatment having more effect which was proved previously. Yava Kshar was selected as a Kshar ingredient as previous study was performed using Yava-Kshar Pratisaran and this project was the extension of previous study. There were some procedural problem in study like possibility of application to other than diseased site, variability in amount of Kshar, no assurance of shelf life due to hygroscopic nature of Kshar and needed intervention of doctor. To overcome the above problems there was need to bring the Kshar in new dosages form i.e. Ointment. Hence some remolding of established modality of treatment was done in present study. To make it user friendly and less complicating, new drug dosage formation i.e. ointment in different percentage of Yava Kshar and different bases was included in this study. Observations noted were analyzed using confidence limit which was fixed at $95 \%$ and the level of significance which was at $5 \%$. Paired and unpaired ' $t$ ' test was applied for objective parameter and chi square test was applied for subjective parameter and ANOVA test was applied for comparison of effect of all ointments. Total 219 well diagnosed patients suffering from $\mathrm{II}^{\circ}$ internal haemorrhoids, not responding to conservative treatment were included in the study. The maximum incidence of Arsha in men of age 25 to 55 years was observed and only 31 female were participated in the trial. The less number of female patients could be due to ignorance and shyness. The male sex prevalence may be due to haemorrhoid disease precipitating dietary habitat and addictions. With reference to diet, those patients who were taking nonvegetarian and spicy diet were more sufferer than patient taking vegetarian and non-spicy diet. This may because of non-vegetarian spicy diet which is prone to develop constipation and increases intra-rectal resting pressure and provoking engorgement of the haemorrhoidal vessels. 
Constipated hard stool having maximum friction to the anal, congested haemorrhoidal mass and produces symptomatic mass and produces symptomatic haemorrhoids. Regularization of the dietary habit along with avoidance of constipation and friction by stool softening agent has their own importance. Therefore, identical ideal dietary regimen and stool softening agents advised in all the groups. The patients were also scrutinized according to 'Habitat'. This observation shows that maximum patients were having same type of habitat and it was interpreted that these patients were more prone to develop internal haemorrhoid.

\section{Degree of $\mathbf{P} / \mathbf{R}$ Bleeding}

\section{On Day 0}

All group had nearly same mean of $\mathrm{P} / \mathrm{R}$ bleeding.

\section{On $3^{\text {rd }}$ Day}

Mean change in $\mathrm{P} / \mathrm{R}$ bleeding occurred in group Yava Kshar ointment 10\% (base Siktha Tailam), Yava Kshar ointment 10\% (base Petroleum Jelly) and Yava Kshar ointment 5\% (base Siktha Tailam). Mean change in $\mathrm{P} / \mathrm{R}$ bleeding was maximum in group treated with Yava Kshar ointment $10 \%$ (base Siktha Tailam), very slight change occurred in groups treated with Yava Kshar ointment 5\% (base Petroleum Jelly), Faktu ointment, Trifgol, Petroleum Jelly and Siktha Tailam. Maximum P/R bleeding was in group treated with Petroleum jelly.

\section{On $10^{\text {th }}$ Day}

Decrease in $\mathrm{P} / \mathrm{R}$ bleeding was observed in group Yava Kshar ointment 5\% (base petroleum jelly), Yava Kshar ointment 5\% (base Siktha Tailam), Yava Kshar ointment 10\% (base Siktha Tailam), Yava Kshar ointment 10\% (base petroleum jelly) and Faktu ointment. P/R bleeding was minimal in Yava Kshar ointment 10\% (base Siktha Tailam) and then in Yava Kshar ointment 5\% (base Siktha Tailam). Slight decrease was observed in Trifgol. There was increase in $\mathrm{P} / \mathrm{R}$ bleeding in group treated with Siktha Tailam and Petroleum jelly.

\section{On $17^{\text {th }}$ Day}

Negligible P/R bleeding was observed in groups treated with Yava Kshar ointment 5\% (base petroleum jelly), Yava Kshar ointment 5\% (base Siktha Tailam), Yava Kshar ointment 10\% (base Siktha Tailam) and Yava Kshar ointment $10 \%$ (base petroleum jelly). Slight P/R bleeding observed in patients treated with Faktu ointment. Very slight decrease in $\mathrm{P} / \mathrm{R}$ bleeding was in groups Trifgol, Siktha Tailam and Petroleum jelly. This may be due to softening of stools.

\section{On $24^{\text {th }}$ day}

No $\mathrm{P} / \mathrm{R}$ bleeding reported by patients of group Yava Kshar ointment 5\% (base petroleum jelly). Yava Kshar ointment 5\% (base Siktha Tailam), Yava Kshar ointment 10\% (base Siktha Tailam) and Yava Kshar ointment 10\% (base petroleum jelly). Incidence of $\mathrm{P} / \mathrm{R}$ bleeding was minimal in group treated with Faktu ointment. Negligible changes in P/R bleeding occurred in group Trifgol, Siktha Tailam, Petroleum jelly, in which decrease was more in group Trifgol.

The probable mode of action of the drug may be due to corrosive action. As 'Yava Kshar' i.e. potash alkali, reduces bleeding and stabilizes vascular endothelium by its chemical cauterization effect. This action may help to control P/R bleeding which is the most important clinical feature of the internal haemorrhoid ${ }^{10}$.

\section{Degree of hemorrhoids}

\section{Till $17^{\text {th }}$ day}

No change in degree of haemorrhoid was observed in all groups.

\section{$24^{\text {th }}$ day}

The changes were observed in group Yava Kshar ointment 5\% base Siktha Tailam, Yava Kshar ointment $10 \%$ base petroleum jelly and Yava Kshar ointment 10\% base Siktha Tailam. Maximum changes were observed in Yava Kshar ointment $10 \%$ base Siktha Tailam. No changes observed in groups Yava Kshar ointment 5\% base petroleum jelly, Faktu ointment, Trifgol powder, plane Siktha Tailam and plane petroleum jelly. Though the difference in degree of haemorrhoid was not found to the marked extent, some other local effects were found which were supposed to support the haemorrhoidal plexuses and not to prolapsed haemorrhoid. The fibrotic (blackish in colour) changes were observed at the site of haemorrhoid, which were recorded in photographs. It may be due to chemical cauterization. In delayed follow ups, it was observed that, the size of haemorrhoid was reduced but not reduced totally. May be after few months, it changes to normal mucosa. Hence it is effective for internal hemorrhoid. Prolapsing haemorrhoidal mass and $\mathrm{P} / \mathrm{R}$ bleeding due to which there was decreased chances of cloth spoilages and improvement of local hygiene by Seitz bath also. Due to decrease in Prolapsing mass overall psychological disturbance of the sufferer was also minimizes as the patient becoming symptoms free. At the same time having tikshna, ushna properties along with katu rasa causes 'Lekhan, Ksharan and Kshapan' i.e. scrubbing of the tissue and decreases size of haemorrhoid. This action may helps to reduce the size of prolapsing haemorrhoid mass ${ }^{10}$.

\section{Assessment of Drug Dose}

Different concentration of Yava Kshar in different ointment was helpful in accessing proper dose of Yava Kshar for local application with reducing complications. It was observed that Group having Yava Kshar 10\% had good results.

\section{Assessment of Different Bases}

Different bases were used i.e. Natural base (Siktha Tailam) and synthetic base (Petroleum jelly). These helped in accessing utility of small dose of Kshar in different bases. As both these bases were tried as a control group and didn't show any significant action, it can be said that action was due to Yava Kshar and not due to bases. Yava Kshar ointment 5\% (base Siktha Tailam), Yava Kshar ointment 10\% (base petroleum jelly), Yava 
Kshar ointment 10\% (base Siktha Tailam) are statistically significant in reducing degree of Haemorrhoid. Moreover it was found that Yava Kshar ointment 10\% having base Siktha Tailam was most effective than other ointments. Because of more concentration of Yava Kshar and more solubility of Siktha Tailam comparative to Petroleum jelly and by medicinal scrubbing (i.e. Lekhan, Ksharan and Kshapan) property of Yava Kshar. Faktu ointment was less effective in reducing degree of haemorrhoids. Finally, all Yava Kshar ointments cure P/R bleeding within less time i.e. 3 to 7 days as compared to Faktu ointment i.e. to 14days.

\section{CONCLUSION}

The $\mathrm{P} / \mathrm{R}$ bleeding was decreased significantly in each group $(\mathrm{p}<0.05)$ except in simple Siktha Tailam and simple Petroleum jelly. The Faktu ointment and Trifgol required 10 days approximately to reduce the $\mathrm{P} / \mathrm{R}$ bleeding where as other all Yava Kshar ointment required 3 days approximately to reduce $\mathrm{P} / \mathrm{R}$ bleeding and it was highly significant $(\mathrm{p}<0.001)$. Though the difference in degree of haemorrhoid was not found to the marked extent however some other local effects were found, which were supposed to give a strong support to the haemorrhoidal plexuses and not to prolapsed haemorrhoid. The fibrotic (blackish in colour) changes were observed at the site of haemorrhoid, which were recorded in photographs. It may be due to chemical cauterization. In delayed follow ups, it was observed that, the size of haemorrhoid was reduced significantly but not totally. May be after few months, it changes to normal mucosa. Hence it is effective for internal haemorrhoid. It was concluded that the application of Yava Kshar ointment of $10 \%$ having base Siktha Tailam was more effective than other ointment (i.e. Yava Kshar ointment 5\% base Siktha Tailam, Yava Kshar ointment 5\% base petroleum jelly, Yava Kshar ointment
$10 \%$ base petroleum jelly, Faktu ointment, Trifgol powder, plane Siktha Tailam and plane Petroleum jelly) in reducing the $\mathrm{P} / \mathrm{R}$ bleeding markedly within minimal period and in reducing the degree of haemorrhoids up to certain extent.

\section{REFERENCES}

1. Trikamji Y. Editor, Nibandhasangraha Sanskrit commentary, In Sushrut Samhita, Sutrasthana, Chapter 33 verse $4,5^{\text {th }}$ ed, Varanasi, India. Chaukhambha Orientalia publications; 1992. p. 144

2. Jha V. Editor, In Amarakosha, Part 2, Manushya Varga, verse 54, New Delhi, India. Motilal Banarasidas publications, Amar Kosha $2^{\text {nd }}$ part Manushya Varga; p. 220

3. Sharma HN. Editor, In Ashtanga Hridaya, Sutrasthana Chapter 13, verse 1-3, $1^{\text {st }}$ ed, Varanasi, India. Chaukhambha Vishwabharati publications; 1996. p. 99

4. http://medical-dictionary.thefreedictionary.com/Haemorrhoids on accessed on 30.05.2013.

5. Shastri K, Chaturvedi G. Editor, Vidyotini hindi commentary. In Charak Samhita part 1, Sutrasthana, Chapter 14, verse 18-19, $1^{\text {st }}$ ed, Varanasi, India. Chaukhambha Bharati Academy publications; 2007. p. $422-423$

6. Shastri K, Chaturvedi G. Editor, Vidyotini hindi commentary. In Charak Samhita part 1, Sutrasthana, Chapter 14, verse 33, $1^{\text {st }}$ ed, Varanasi, India. Chaukhambha Bharati Academy publications; 2007. p. 425

7. Ukhalkar VP. To study the efficacy of Pratisarniya Yava Kshar in Management of Abhyantar Gudarsha, Dissertation for M.S. (Shalya), S.R.T.M.U. Nanded; 2007.

8. Tripathi B. Editor, Dipika hindi commentary. In Sharangadhara Samhita, Madhayama Khanda Chapter 11 , verse $102-105,1^{\text {st }}$ ed Varanasi, India. Chaukhambha Surabharati publications; 2006. p. 282

9. Sharma S. Rasatarangini, $11^{\text {th }}$ ed; Chapter 6, Verse 56-63, Varanasi, Motilal Banarasidas publications; 2004. p. 114-15

10. Chunekar KC, Pandey GS. Editor, Bhavaprakash Nighantu, Haritakyadi Varga, verse 29-30, $1^{\text {st }}$ ed, Varanasi, India. Chaukhambha Bharti Academy; 2006. p. 641

Cite this article as:

Ukhalkar V. P. Evaluation of effects of Yava kshar ointment on Abhyantar arsha (internal piles). Int. J. Res. Ayurveda Pharm. 2013;4(4):574-582 http://dx.doi.org/10.7897/2277-4343.04425 